\title{
Analysis of Mono-ADP-Ribosylation Levels in Human Colorectal Cancer
}

This article was published in the following Dove Press journal:

Cancer Management and Research

\section{Chuan-Ling Wang $(\mathbb{D}$ \\ Yi Tang \\ Ming Li \\ Ming Xiao \\ Qing-Shu Li \\ Lian Yang \\ Xian Li \\ Ling Yin \\ Ya-Lan Wang}

Department of Pathology, Molecular Medicine and Cancer Research Center. Chongqing Medical University,

Chongqing, 4000I6, People's Republic of China
Correspondence: Ya-Lan Wang Department of Pathology, Molecular Medicine and Cancer Research Center, Chongqing Medical University, Chongqing, 400016, People's Republic of China

Tel $+86 \quad 13628308360$

Fax +86 2368485111

Email wangyalan@cqmu.edu.cn
Background: Colorectal cancer remains a major public health problem with high morbidity and mortality rates. In the search for the mechanisms of colorectal cancer occurrence and development, increasing attention has been focused on epigenetics. The overall level of Mono-ADP-ribosylation, an epigenetic, has not been investigated now. The aim of our study was to analysis of the overall level of mono-ADP-ribosylation in colorectal cancer.

Methods: Immunohistochemistry was used to investigate the level of mono-ADPribosylation in colorectal cancer and normal colorectal adjacent tissue from 64 CRC patients. The data of patient demographic, clinical and pathological characteristics were acquired and analyzed.

Results: Mono-ADP-ribosylation was present in both colorectal adenocarcinoma and normal colorectal tissue. The overall level of mono-ADP-ribosylation in colorectal cancer was significantly higher than that in normal colorectal adjacent tissue. In the nucleus, the majority of samples in the high-level group were colorectal adenocarcinoma (55/64), but the opposite was true for normal colorectal tissues (7/32). In particular, increases in the level of monoADP-ribosylation in the cytoplasm of colorectal cancer cells was associated with a greater invasion depth of the tumor.

Conclusion: The increased level of mono-ADP-ribosylation in colorectal cancer enhances tumor invasion, which suggests that mono-ADP-ribosylation is involved in the development of colorectal cancer and may become a new direction to solve the problem of colorectal cancer.

Keywords: mono-ADP-ribosylation, colorectal cancer, immunohistochemistry

\section{Introduction}

Colorectal cancer (CRC) is one of the most common malignancies of the digestive tract, with the third highest incidence and the second highest mortality rate in the world, ${ }^{1}$ and is a serious threat to human health. According to the American Cancer Society, in 2020, the number of cases of newly diagnosed CRC is estimated to be 147,950, and approximately 53,200 people will die of CRC in the United States. ${ }^{2}$ Additionally, the incidence and mortality of CRC in China is expected to increase to 642,300 and 221,100 by $2025 .^{3}$ Therefore, elucidating the etiology of CRC and identifying pathogenesis and carcinogenic factors are of great significance to establish new early diagnosis methods, implement effective preventive measures and improve treatment.

With the development of proteomics, protein post-translational modifications (PTMs) and their effects on protein function have been further studied with respect to the occurrence and development of CRC. ADP ribosylation is a PTM involving 


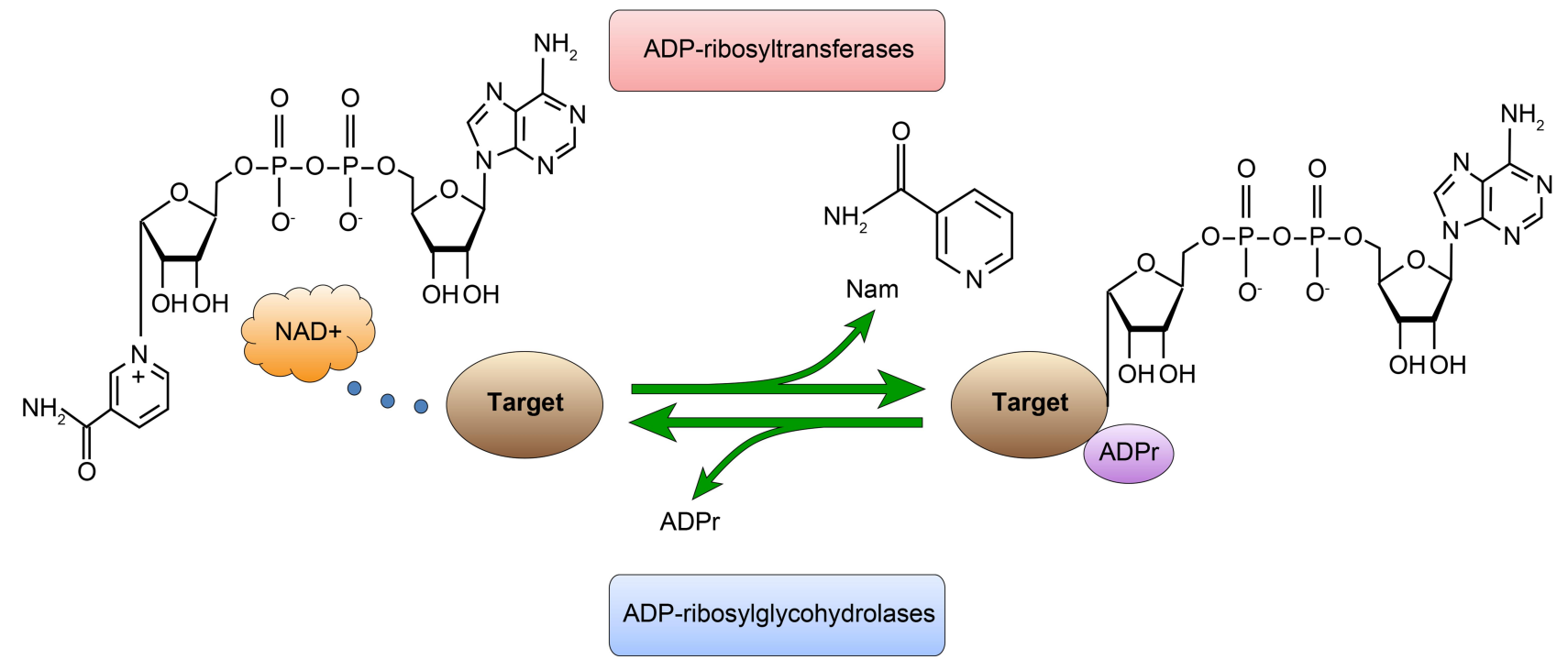

Figure I The process of mono-ADP-ribosylation (MARylation). ADP-ribosylation is reversible; it is catalyzed by ADP-ribosyltransferases (ARTs) and removed by ADPribosylglycohydrolases.

the covalent binding of an ADP-ribose moiety from nicotinamide adenine dinucleotide $\left(\mathrm{NAD}^{+}\right)$to amino acid residues of target proteins by ADP-ribosyltransferases (Figure 1). Different residues can be covalently modified, which can produce different signals in regulating protein functions and interactions. ADP ribosylation can occur as mono- or poly-ADP-ribosylation, which are reversible PTMs that participate in cell signal transduction, DNA repair and gene regulation. ${ }^{4}$

Although great progress has been made in the study of ADP ribosylation in recent years, the identification of ADP-ribosylated proteins, chromatin binding sites and amino acid targets has been challenging. The role of polyADP-ribosylation (PARylation) has been widely studied in the past few decades, but the physiological function of mono-ADP-ribosylation (MARylation) is still poorly understood. However, an increasing number of studies has shown that MARylation is related to a variety of cellular processes, including immune regulation, endoplasmic reticulum stress and cell metabolism. ${ }^{4,5}$ This study will assess the level of MARylation in CRC specimens and explore the characteristics of the specimens.

\section{Materials and Methods}

\section{Patients and Tumor Specimens}

Paired tumor and distal normal tissues were collected from 64 CRC patients at the First Affiliated Hospital of Chongqing Medical University. The study was approved by the Clinical Research Ethics Committee of Chongqing Medical University. All subjects provided written informed consent. The age of the patients ranged from 31 to 85 years old with a median age of 60.5 years old.

\section{Immunohistochemistry}

Immunohistochemical staining was completed using a standard streptavidin peroxidase kit (Maixin Biotechnologies, Fuzhou, China) on $4 \mu \mathrm{m}$ sections from paraffin-embedded colorectal carcinoma tissues. In short, all sections were baked at $60^{\circ} \mathrm{C}$ for 2 hours, deparaffinized with xylene and dehydrated with a graded series of ethanol. Then, the sections were sequentially treated with citrate buffer $(10 \mathrm{mM}, \mathrm{pH} 6.0)$ in a pressure chamber for antigen retrieval. Blocking reagents were used to reduce endogenous peroxidase activity and nonspecific staining. Then, rabbit anti-mono-ADP-ribose primary antibody (1: 300, Millipore, Billerica, MA, USA) was added to the sections and incubated at $4{ }^{\circ} \mathrm{C}$ overnight. The following day, the sections were incubated in biotin-labeled secondary antibody for $30 \mathrm{~min}$ at $37^{\circ} \mathrm{C}$ before they were treated with streptavidin peroxidase. Antibody binding in all the sections was visualized using a diaminobenzidine (DAB) Chromogen Kit (Maixin Biotechnologies, Fuzhou, China).

\section{Evaluation}

According to the World Health Organization criteria (2019, fifth edition), ${ }^{6}$ two independent and experienced pathologists who were blinded to the patients' clinical data judged 
the differentiation grade, invasive depth, histological classification, TNM stage and lymph node metastasis of the tumor and scored the immunohistochemistry results using a specific method based on previous literature. ${ }^{7}$ The staining intensity score was graded as 0 , no staining; 1 , light yellow; 2 , brown; and 3, dark brown. The proportion of positive cells in 100 tumor cells was observed by microscopy, and the cell positive rate scores were recorded as follows: $0,0 \sim 5 \% ; 1$, $6 \% \sim 25 \% ; 2,26 \% \sim 50 \% ; 3,51 \% \sim 75 \%$; and $4,>75 \%$. The intensity and positive rate scores were added to produce the final score, which was classified as follows: $<2$, negative (-); $2 \sim 3$, slight positive $(+) ; 4 \sim 5$, moderately positive $(++) ; 6 \sim 7$, strongly positive $(+++)$. For the analysis, the specimens were divided into two groups: negative and slight positive were defined as the low-level group, whereas moderately positive and strongly positive were defined as the high-level group.

\section{Statistical Analysis}

Statistical analysis was performed using SPSS 19.0 software. Differences between different groups were analyzed using the chi-square and Wilcoxon tests. A $P$ value less than $0.05(\mathrm{P}<0.05)$ was considered statistically significant.

\section{Results}

Immunohistochemistry staining showed that MARylation was present in both colorectal adenocarcinoma and normal colorectal tissue (Figures 2 and 3). The level of MARylation in colorectal adenocarcinoma was significantly higher than that in normal colorectal tissue $(\mathrm{P}<$ $0.05)$ (Table 1) in both the nucleus and cytoplasm $(\mathrm{P}<$ 0.05 ) (Tables 2 and 3). In the nucleus, the majority of samples in the high-level group were colorectal adenocarcinoma (55/64), but the opposite was true for normal colorectal tissues (7/32) (Figure 3, Table 2). Significantly, no high levels of MARylation were observed in the cytoplasm of normal colorectal tissue $(0 / 32)$.

The association of clinicopathological characteristics and MARylation staining of the nucleus and cytoplasm in colorectal adenocarcinoma was analyzed. It was found that positive MARylation was not related to sex, age, location, lymph node metastasis, differentiation grade or TNM stage, but positive MARylation in the cytoplasm was related to the depth of invasion $(\mathrm{P}<0.05)$; in the nucleus, the positive MARylation was associated with the histological type of CRC $(\mathrm{P}<0.05)$ (Table 4).

The above two distinct clinicopathological features were further studied. The level of MARylation was gradually enhanced with increasing invasion depth $(\mathrm{P}<0.05)$ (Table 5). The level of MARylation in the nucleus was not significantly different between colorectal adenocarcinoma and mucinous adenocarcinoma (Table 6).

\section{Discussion}

MARylation has been shown to be involved in different pathophysiological processes, such as signal transduction, gene transcription, stress response, DNA damage repair and apoptosis, ${ }^{4,5,8}$ by regulating the interaction of the target with other molecules or changing the activity of the target if the target is an enzyme. Due to the limitations of technology, previous studies on the role of MARylation in CRC were mainly focused on various enzymes, ${ }^{9-11}$ which could not identify the specific modification targets. However, it has been determined that nonenzymatic proteins, DNA and RNA can undergo MARylation. To clarify the role of MARylation, the level of MARylation was detected by immunohistochemistry in colorectal adenocarcinoma and normal colorectal tissues for the first time. Compared with normal colorectal tissues, CRC tissues showed significantly increased MARylation in the
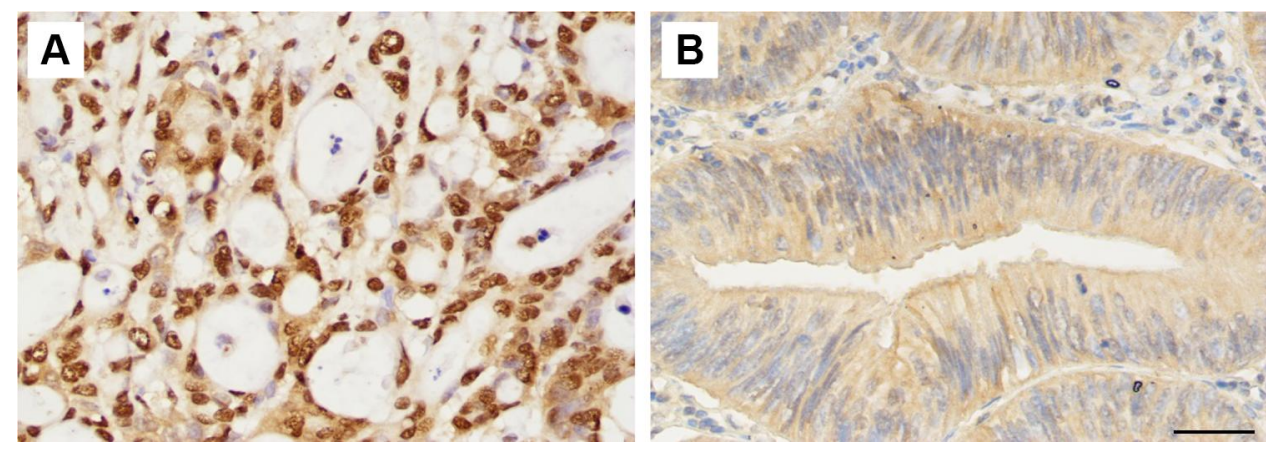

Figure 2 Staining of mono-ADP-ribose binding reagent in colorectal adenocarcinoma tissue samples. $($ A $)$ Strongly positive staining $(\times 400)$. $($ B $)$ Slight positive staining $(\times 400)$. Scale bar $=50 \mu \mathrm{m}$. 


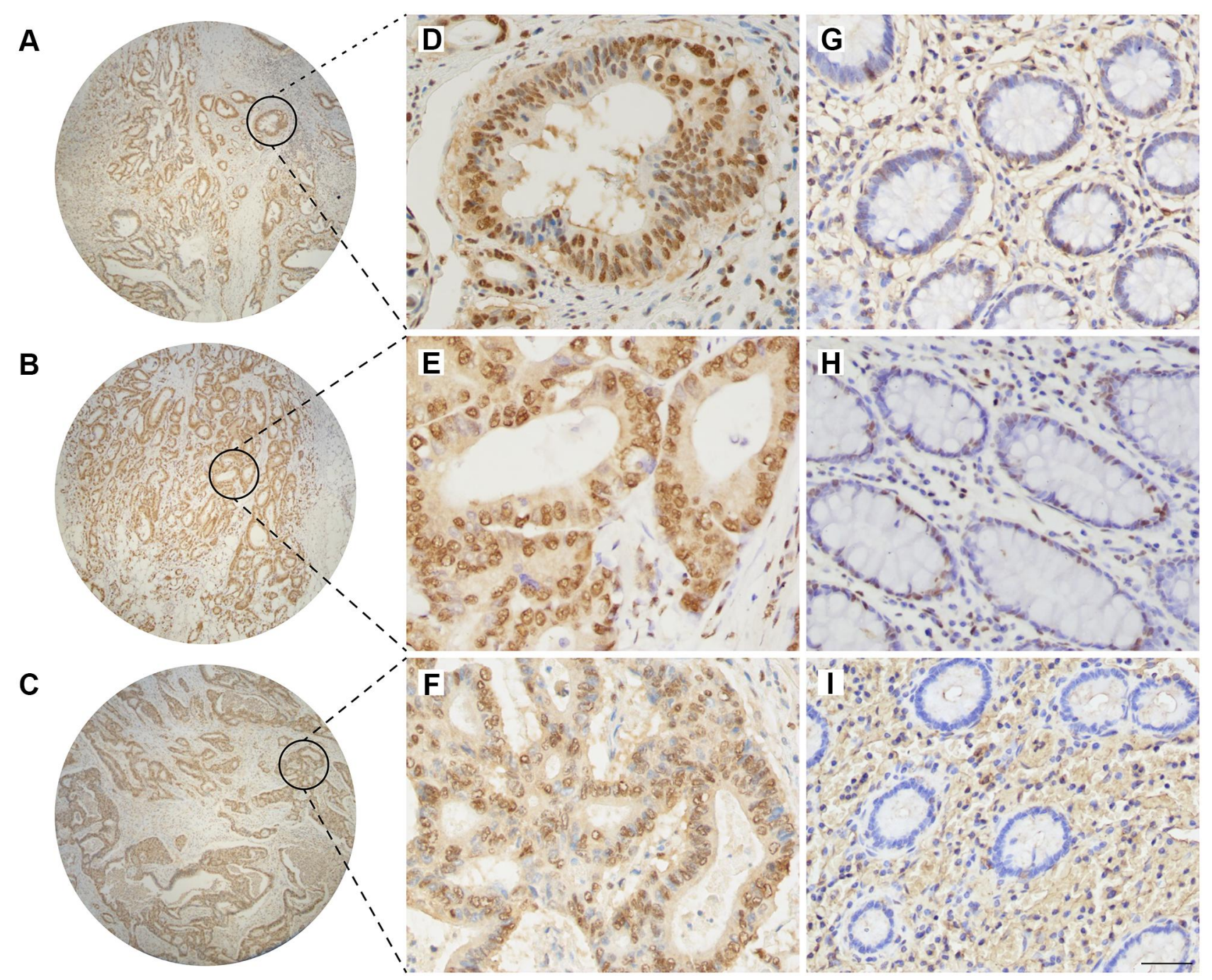

Figure 3 The level of mono-ADP-ribose binding reagent in colorectal adenocarcinoma and normal colorectal tissues. Positive staining of mono-ADP-ribose binding reagent in colorectal adenocarcinoma $(\mathbf{A}-\mathbf{C})(\times 4),(\mathbf{D}-\mathbf{F})(\times 400)$. Positive staining of mono-ADP-ribose binding reagent in normal colorectal tissue $(\mathbf{G}-\mathbf{I})(\times 400)$. Scale bar=50 $\mu \mathrm{m}$.

cytoplasm and nucleus. In particular, with the increase in the level of MARylation in the cytoplasm, the depth of invasion became increasingly deeper, which suggested that MARylation was involved in the progression of CRC.

High levels of MARylation in the cytoplasm of CRC cells promoted tumor invasion, which may be related to the

Table I Different Degrees of Mono-ADP-Ribose Binding Reagent Staining in Colorectal Adenocarcinoma and Normal Colorectal Tissue

\begin{tabular}{|l|c|c|c|c|}
\hline & N & Low & High & $P$ \\
\hline Colorectal adenocarcinoma & 64 & I & 63 & $<0.00$ I* \\
\hline Normal colorectal tissue & 32 & 25 & 7 & \\
\hline
\end{tabular}

Note: *Signifies $\mathrm{P}<0.05$.

Abbreviations: N, number; Low, low-level group; High, high-level group. different effects of modified proteins. The shedding of tumor cells from the primary site to the surrounding environment is a multistep process in which cell adhesion is the key step. $\beta$-Catenin and cell surface E-cadherin interact to form a cell-adhesion junction complex that mediating intercellular adhesion, which is closely related to tumor invasion and

Table 2 Mono-ADP-Ribose Binding Reagent in the Nucleus of Colorectal Adenocarcinoma and Normal Colorectal Tissue

\begin{tabular}{|l|c|c|c|c|}
\hline \multirow{2}{*}{} & \multicolumn{4}{|c|}{ Nucleus } \\
\cline { 2 - 5 } & N & Low & High & P \\
\hline Colorectal adenocarcinoma & 64 & 9 & 55 & $<0.00 I^{*}$ \\
\hline Normal colorectal tissue & 32 & 25 & 7 & \\
\hline
\end{tabular}

Note: *Signifies $\mathrm{P}<0.05$.

Abbreviations: N, number; Low, low-level group; High, high-level group. 
Table 3 Mono-ADP-Ribose Binding Reagent in the Cytoplasm of Colorectal Adenocarcinoma and Normal Colorectal Tissue

\begin{tabular}{|l|c|c|c|c|}
\hline \multirow{2}{*}{} & \multicolumn{4}{|c|}{ Cytoplasm } \\
\cline { 2 - 5 } & N & Low & High & P \\
\hline Colorectal adenocarcinoma & 64 & 7 & 57 & $<0.00 I^{*}$ \\
Normal colorectal tissue & 32 & 32 & 0 & \\
\hline
\end{tabular}

Note: *Signifies $\mathrm{P}<0.05$.

Abbreviations: $\mathrm{N}$, number; Low, low-level group; High, high-level group.

metastasis. Previous studies have shown that the MARylation of glycogen synthase kinase $3 \beta$ (GSK-3 $\beta$ ) can suppress its kinase activity, ${ }^{12}$ which is responsible for the phosphorylation and consequent degradation of $\beta$-catenin. MARylation of GSK3 $\beta$ leads to the accumulation of $\beta$ catenin in the nucleus and activation of downstream target
Table 5 The Difference Analysis of Invasive Depth and the Level of Mono-ADP-Ribose Binding Reagent in Cytoplasm

\begin{tabular}{|l|c|c|c|c|}
\hline Invasive Depth & N & Low & High & P \\
\hline Submucosa & 2 & I & I & 0.049* \\
\hline Muscularis & 7 & 2 & 5 & \\
\hline Serosa and adventitia & 55 & 4 & 51 & \\
\hline
\end{tabular}

Note: *Signifies $\mathrm{P}<0.05$.

Abbreviations: N, number; Low, low-level group; High, high-level group.

genes such as c-myc, MMP-7 and CyclinD1. Aromatic hydrocarbon receptor (AHR), a component of the E3 ubiquitin ligase, can ubiquitinate $\beta$-catenin to mark it for degradation. Colonic tumors were frequently observed in AHR-/- mice, ${ }^{13}$ and the MARylation of AHR inhibited its activity, ${ }^{14}$ resulting in a reduction in ubiquitination.

Table 4 Comparison of Clinicopathological Characteristics in 64 Cases with Colorectal Adenocarcinoma

\begin{tabular}{|c|c|c|c|c|}
\hline Clinicopathological Features & No. of Patients $(n=64)$ & Positivity Rate (\%) & $P$ (Cytoplasm) & $P$ (Nucleus) \\
\hline Age & & & 0.257 & 0.857 \\
\hline$<60$ & 28 & $43.75 \%$ & & \\
\hline$\geq 60$ & 36 & $56.25 \%$ & & \\
\hline Sex & & & 0.439 & 0.713 \\
\hline Males & 40 & $62.50 \%$ & & \\
\hline Females & 24 & $37.50 \%$ & & \\
\hline Location & & & 0.662 & 0.261 \\
\hline Left colon & II & $17.74 \%$ & & \\
\hline Right colon & 17 & $27.42 \%$ & & \\
\hline Rectum & 34 & $54.84 \%$ & & \\
\hline Lymph node metastasis & & & 0.272 & 0.194 \\
\hline No & 35 & $54.69 \%$ & & \\
\hline Yes & 29 & $45.31 \%$ & & \\
\hline Invasive depth & & & $<0.00 I^{*}$ & 0.847 \\
\hline Submucosa & 2 & $3.13 \%$ & & \\
\hline Muscularis & 7 & $10.94 \%$ & & \\
\hline Serosa and adventitia & 55 & $85.94 \%$ & & \\
\hline Differentiation grade & & & 0.227 & 0.238 \\
\hline Low grade & 26 & $40.63 \%$ & & \\
\hline High grade & 38 & $59.37 \%$ & & \\
\hline TNM stage & & & 0.472 & 0.321 \\
\hline I & 7 & $10.94 \%$ & & \\
\hline II & 26 & $40.63 \%$ & & \\
\hline III & 22 & $34.38 \%$ & & \\
\hline IV & 9 & $14.06 \%$ & & \\
\hline Histological classification & & & 0.667 & $0.001 *$ \\
\hline Adenocarcinoma & 54 & $84.38 \%$ & & \\
\hline Mucinous adenocarcinoma & 10 & $15.63 \%$ & & \\
\hline
\end{tabular}

Note: *Signifies $\mathrm{P}<0.05$. 
Table 6 The Difference Analysis of Histological Classification and the Level of Mono-ADP-Ribose Binding Reagent in Nucleus

\begin{tabular}{|l|c|c|c|c|}
\hline Histological Classification & N & Low & High & P \\
\hline Adenocarcinoma & 54 & 4 & 50 & 0.78 I \\
\hline Mucinous adenocarcinoma & 10 & I & 9 & \\
\hline
\end{tabular}

Abbreviations: N, number; Low, low-level group; High, high-level group.

Ubiquitination mediated by ubiquitin E3 ligase $\beta$-transducin repeat containing protein $1(\beta-\operatorname{TrCP} 1)$ is another $\beta$-catenin degradation pathway. ${ }^{15}$ MARylation of $\beta$-TrCP1 can upregulate its expression. ${ }^{16}$ Karyopherin- $\beta 1$ (KPNB1) can transport proteins, such as $\beta$-catenin and NF-kB p65, or RNAs into and out of the nucleus through nuclear pore complexes. ${ }^{17,18} \mathrm{At}$ present, the effect of MARylation on KPNB1 function is unclear, but it is possible that MARylation inhibits its carrier function and affects KPNB1-assisted nuclear-cytoplasmic transport protein. ${ }^{19}$ Recent studies have indicated that
MARylation of Golgin-97 is necessary to ensure the correct transport of E-cadherin from the reverse Golgi apparatus to the plasma membrane. ${ }^{20}$ Inhibition of MARylation will prevent Golgin-97-controlled E-cadherin from reaching the plasma membrane and promote epithelial-mesenchymal transformation (EMT). Therefore, MARylation of different proteins in the cytoplasm causes the aggregation of intracellular $\beta$-catenin, a reduction in the binding of $\beta$-catenin to E-cadherin at the cell membrane, and the decreased distribution of E-cadherin to the plasma membrane, all of which lead to decreased adhesion between cells and an increased likelihood that tumor cells will infiltrate the surrounding microenvironment.

In addition, three key proteins in the cytoplasm involved in endoplasmic reticulum stress can be affected by MARylation, which possibly influences the invasion of tumor cells. MARylation of inositol-requiring enzyme-1 $\alpha$ (IRE-l $\alpha$ ) and protein kinase RNA-like endoplasmic reticulum kinase (PERK) can increase their kinase activity and

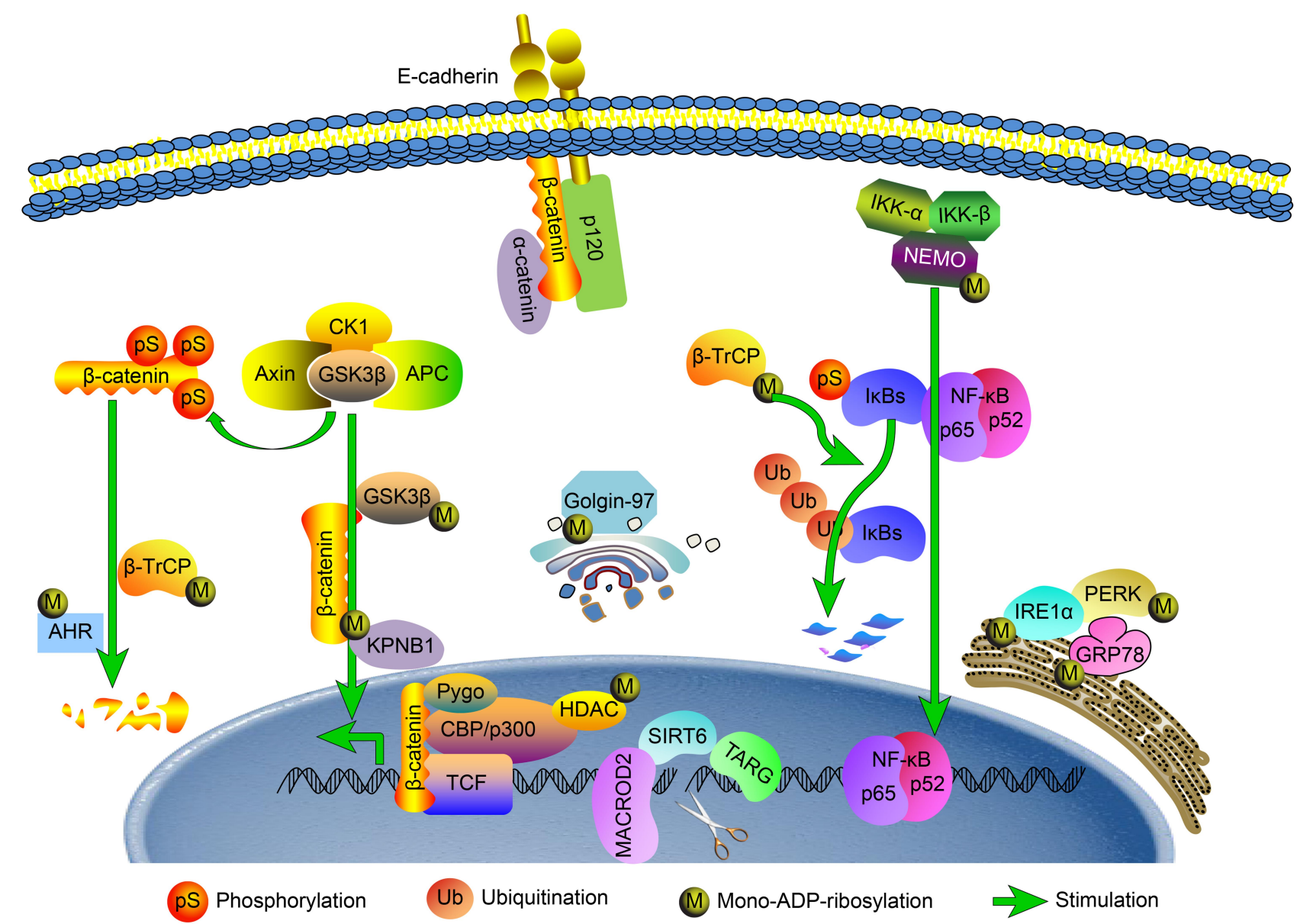

Figure 4 Schematic diagram of how MARylation is predicted to be involved in the regulation of colorectal cancer. The possible mechanisms of MARylation in colorectal cancer are described with regard to endoplasmic reticulum stress, DNA damage repair, EMT, $\beta$-catenin activity and the NF- $\mathrm{KB}$ signaling pathway. 
stimulate downstream signaling, ${ }^{21}$ additionally, activating PERK promotes tumor invasion. ${ }^{22}$ Meanwhile, MARylation of glucose-regulated protein 78 (GRP78) results in its inactivation, ${ }^{23}$ while silencing GRP78 in colon cancer can reduce E-cadherin expression, promote EMT, and facilitate tumor invasion. ${ }^{24}$ These studies are consistent with the observed effect of MARylation on tumor invasion, but the proteins modified by MARylation in $\mathrm{CRC}$ need to be identified more readily.

In this study, MARylation of normal colorectal tissues was mainly localized to the nucleus, with the majority of cytoplasm showing none; by contrast, the level of MARylation in the cytoplasm of CRC was significantly increased. MARylation of $\mathrm{NF}-\kappa \mathrm{B}$ essential modulator (NEMO) in the cytoplasm prevents the activation of the NEMO IKK/complex; ${ }^{25}$ this blocks the phosphorylation and ubiquitination of I $\kappa \mathrm{B}$ protein, which then cannot dissociate from $N F-\kappa B$ and inhibits $N F-\kappa B$ activation. However, MARylation of $\beta$-TrCP1 upregulates its expression, and $\beta$-TrCP1 mediates the degradation of $\mathrm{I} \kappa \mathrm{B}$, which releases the $N F-\kappa B$ dimer and activates the $N F-\kappa B$ pathway. $^{26}$ In pancreatic cancer cells, PARP14 is responsible for the nuclear translocation of high levels of phos-

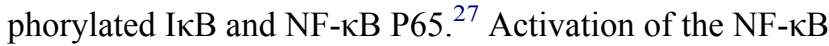
signaling pathway promotes the invasion and survival of CRC cells. ${ }^{28}$ However, MARylation of different proteins in the NF- $\kappa \mathrm{B}$ signaling pathway has an inverse effect on pathway activation, and the effects of MARylation on CRC have not been clearly defined. Because modification is a reversible and dynamic process, the specific indicators of MARylation in CRC need to be further clarified.

The level of MARylation in the nuclei of CRC cells was significantly higher than that in normal colorectal cells. Compared with colorectal adenocarcinoma, mucinous adenocarcinoma progresses more rapidly and has a worse prognosis. Although the positive proportion of MARylation in the nucleus was related to histological type, there was no significant difference in MARylation level between colorectal adenocarcinoma and mucinous adenocarcinoma. Our previous studies have shown that MARylation of H3R117 in LOVO cells is involved in the regulation of oncogene methylation and promotes tumor survival, which may contribute to the development of CRC by affecting the structure and dynamics of chromatin. ${ }^{29,30}$ In addition, MARylation of histone deacetylase 2 (HDAC2) and HDAC3 enables them to dissociate from the gene promoter, and the binding of transcription factor $\mathrm{P} 300$ to the gene promoter can enhance the expression of downstream genes that promote antiapoptotic activity in tumor cells. ${ }^{31}$ MARylation of cell cycle checkpoint kinase 1 (Chk1) hinders the phosphorylation of Chk1S345, and the removal of MARylation leads to mitotic signaling defects. ${ }^{32}$ In addition, MARylation of the hydrolases PARG, ${ }^{33}$ TARG $1,{ }^{34}$ MACROD2 $2^{35}$ and PARP1 $1^{36}$ shifts their activities toward cell damage repair. During the repair process, the export of MACROD2 from the nucleus to the cytoplasm increases. ${ }^{37}$ Although its target is not clear, this dynamic regulation may vary according to the modification requirements of different targets for the cytoplasm and nucleus when cell damage occurs.

Current research suggests that the left and right colons have different embryonic origins and different physiological and molecular backgrounds; these differences can thus influence the choice of treatment. ${ }^{38}$ However, in this study, no difference in MARylation was observed in CRC originating from different parts of the colon, and the significantly increased level of MARylation in CRC may have nothing to do with the originating location.

In summary, MARylation may be involved in many aspects of the development of CRC (Figure 4). Although the specific targets of this modification are not clear, the overall modification level of CRC is much higher than that of normal tissues. Unfortunately, the detection techniques and methods for MARylation are limited, and the role of MARylation in normal colorectal tissues has not been discussed. Moreover, the role of known targets with monoADP-ribose modifications in CRC requires further investigation. In this study, the overall level of MARylation in CRC was observed for the first time, and the possible mechanisms were discussed, providing new context and information relating to the epigenetics of $\mathrm{CRC}$. In the future, we will continue to study the function of specific site modifications and their effect on CRC.

\section{Ethics Approval}

All of the patients provided informed consent, and the study was conducted in accordance with the Declaration of Helsinki. This study passed the ethical review of the Ethics Committee of Chongqing Medical University.

\section{Funding}

The research was supported by the Innovation Project of Graduate Student in Chongqing (grant no. CYB17100); the Science and Technology Research Foundation of Chongqing Municipal Education Commission (KJQN 
201800435); the Science and Technology Research Foundation of Chongqing Municipal Education Commission (KJQN 201900403); the Innovation Project of Graduate Student in Chongqing (CYB19160); and the Foundation and Cutting Edge Research Project from the Science and Technology Commission of Chongqing (cstc2016jcyjA0233).

\section{Disclosure}

The authors declare that they have no conflicts of interest for this work.

\section{References}

1. Bray F, Ferlay J, Soerjomataram I, Siegel R, Torre L, Jemal A. Global cancer statistics 2018: GLOBOCAN estimates of incidence and mortality worldwide for 36 cancers in 185 countries. CA Cancer J Clin. 2018;68(6):394-424. doi:10.3322/caac.21492

2. Siegel RL, Miller KD, Goding Sauer A, et al. Colorectal cancer statistics, 2020. CA Cancer J Clin. 2020;70(3):145-164. doi:10.33 22/caac. 21601

3. Zhang L, Cao F, Zhang G, et al. Trends in and predictions of colorectal cancer incidence and mortality in china from 1990 to 2025 . Front Oncol. 2019;9:98. doi:10.3389/fonc.2019.00098

4. Butepage M, Eckei L, Verheugd P, Luscher B. Intracellular Mono-ADP-ribosylation in signaling and disease. Cells. 2015;4 (4):569-595. doi:10.3390/cells4040569

5. Luscher B, Butepage M, Eckei L, Krieg S, Verheugd P, Shilton BH. ADPribosylation, a multifaceted posttranslational modification involved in the control of cell physiology in health and disease. Chem Rev. 2018;118 (3):1092-1136. doi:10.1021/acs.chemrev.7b00122

6. Nagtegaal ID, Arends MJ, Odze RD, Ak L. World Health Organization Classification of Tumours of the Colon and Rectum Revised. 5th ed. Lyon: IARC; 2019.

7. Fromowitz F, Viola M, Chao S, et al. ras p21 expression in the progression of breast cancer. Human Pathol. 1987;18 (12):1268-1275. doi:10.1016/s0046-8177(87)80412-4

8. Qi H, Price BD, Day TA. Multiple roles for mono- and poly (ADP-ribose) in regulating stress responses. Trends Gene. 2019;35 (2):159-172. doi:10.1016/j.tig.2018.12.002

9. Raval-Fernandes S, Kickhoefer V, Kitchen C, Rome L. Increased susceptibility of vault poly (ADP-ribose) polymerase-deficient mice to carcinogen-induced tumorigenesis. Cancer Res. 2005;65 (19):8846-8852. doi:10.1158/0008-5472.CAN-05-0770

10. Wu CF, Xiao M, Wang YL, et al. PARP10 influences the proliferation of colorectal carcinoma cells, a preliminary study. Mol Biol. 2020;54 (2):220-228. doi:10.31857/S0026898420020184

11. Tang Y, Wang Y, Yang L, et al. Inhibition of arginine ADP-ribosyltransferase 1 reduces the expression of poly (ADP-ribose) polymerase-1 in colon carcinoma. Int $J$ Mol Med. 2013;32(1):130-136. doi:10.3892/ijmm.2013.1370

12. Feijs K, Kleine H, Braczynski A, et al. ARTD10 substrate identification on protein microarrays: regulation of GSK3 $\beta$ by mono-ADPribosylation. CCS. 2013;11(1):5. doi:10.1186/1478-811X-11-5

13. Kawajiri K, Kobayashi Y, Ohtake F, et al. Aryl hydrocarbon receptor suppresses intestinal carcinogenesis in ApcMin/+ mice with natural ligands. Proc Nat Acad Sci USA. 2009;106(32):13481-13486. doi:10.1073/pnas.0902132106

14. Gomez A, Bindesbøll C, Satheesh S, et al. Characterization of TCDD-inducible poly-ADP-ribose polymerase (TIPARP/ARTD14) catalytic activity. Biochem J. 2018;475(23):3827-3846. doi:10.1042/ BCJ20180347
15. Ougolkov A, Zhang B, Yamashita K, et al. Associations among beta-TrCP, an E3 ubiquitin ligase receptor, beta-catenin, and NF-kappaB in colorectal cancer. $J$ Natl Cancer Inst. 2004;96 (15):1161-1170. doi:10.1093/jnci/djh219

16. Guo T, Zuo Y, Qian L, et al. ADP-ribosyltransferase PARP11 modulates the interferon antiviral response by mono-ADP-ribosylating the ubiquitin E3 ligase $\beta$-TrCP. Nat Microbiol. 2019;4 (11):1872-1884. doi:10.1038/s41564-019-0428-3

17. Liang P, Zhang H, Wang G, et al. KPNB1, XPO7 and IPO8 mediate the translocation ofNF-kB/p65 into the nucleus. Traffic. 2013;14 (11):1132-1143. doi:10.1111/tra.12097

18. Lu T, Bao Z, Wang Y, et al. Karyopherin $\beta 1$ regulates proliferation of

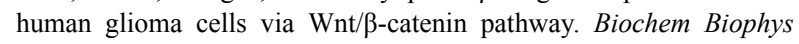
Res Commun. 2016;478(3):1189-1197. doi:10.1016/j. bbrc.2016.08.093

19. Di Girolamo M. Regulation of nucleocytoplasmic transport by ADPribosylation: the emerging role of karyopherin- $\beta 1$ mono-ADPribosylation by ARTD15. Current Topics Microbiol Immunol. 2015;384:189-209. doi:10.1007/82_2014_421

20. Grimaldi G, Schembri L, Monte ML, et al. PARP12-catalyzed mono-ADP-ribosylation of Golgin-97 controls the transport of E-cadherin. bioRxiv. In press 2020. doi:10.1101/2020.05.05.078097

21. Jwa M, Chang P. PARP16 is a tail-anchored endoplasmic reticulum protein required for the PERK- and IRE1 $\alpha$-mediated unfolded protein response. Nature Cell Biol. 2012;14(11):1223-1230. doi:10.1038/ ncb2593

22. Feng Y, Jin D, Sokol E, Reinhardt F, Miller D, Gupta P. Cancerspecific PERK signaling drives invasion and metastasis through CREB3L1. Nat Commun. 2017;8(1):1079. doi:10.1038/s41467-01701052-y

23. Fabrizio G, Di Paola S, Stilla A, et al. ARTC1-mediated ADP-ribosylation of GRP78/BiP: a new player in endoplasmic-reticulum stress responses. Cell Mol Life Sci. 2015;72 (6):1209-1225. doi:10.1007/s00018-014-1745-6

24. Chang YJ, Chen WY, Huang CY, Liu HH, Wei PL. Glucose-regulated protein 78 (GRP78) regulates colon cancer metastasis through EMT biomarkers and the NRF-2/HO-1 pathway. Tumour Biol. 2015;36 (3):1859-1869. doi:10.1007/s13277-014-2788-x

25. Verheugd P, Forst A, Milke L, et al. Regulation of NF- $\mathrm{kB}$ signalling by the mono-ADP-ribosyltransferase ARTD10. Nature Commun. 2013;4:1683. doi:10.1038/ncomms 2672

26. Yaron A, Gonen H, Alkalay I, et al. Inhibition of NF-kappa-B cellular function via specific targeting of the I-kappa-B-ubiquitin ligase. EMBO J. 1997;16(21):6486-6494. doi:10.1093/emboj/16.21.6486

27. Yao N, Chen Q, Shi W, Tang L, Fu Y. PARP14 promotes the proliferation and gemcitabine chemoresistance of pancreatic cancer cells through activation of NF- $\mathrm{BB}$ pathway. Mol Carcinogene. 2019;58(7):1291-1302. doi:10.1002/mc.23011

28. Xie B, Nie S, Hu G, et al. The Involvement of NF-kB/Klotho signaling in colorectal cancer cell survival and invasion. Pathol Oncol Res. 2019;25(4):1553-1565. doi:10.1007/s12253-018-0493-6

29. Li M, Tang Y, Li Q, Xiao M, Yang Y, Wang Y. Mono-ADPribosylation of H3R117 traps 5mC hydroxylase TET1 to impair demethylation of tumor suppressor gene TFPI2. Oncogene. 2019;38 (18):3488-3503. doi:10.1038/s41388-018-0671-8

30. Ling F, Tang Y, Li M, et al. Mono-ADP-ribosylation of histone 3 at arginine-117 promotes proliferation through its interaction with $\mathrm{P} 300$. Oncotarget. 2017;8:72773-72787. doi:10.18632/oncotarget.20347

31. Mehrotra P, Riley J, Patel R, Li F, Voss L, Goenka S. PARP-14 functions as a transcriptional switch for Stat6-dependent gene activation. J Biol Chem. 2011;286(3):1767-1776. doi:10.1074/jbc.M110.157768

32. Wang Z, Grosskurth S, Cheung T, et al. Pharmacological inhibition of PARP6 triggers multipolar spindle formation and elicits therapeutic effects in breast cancer. Cancer Res. 2018;78(23):6691-6702. doi:10.1158/0008-5472.CAN-18-1362 
33. Tanuma S, Shibui Y, Oyama T, Uchiumi F, Abe H. Targeting poly (ADP-ribose) glycohydrolase to draw apoptosis codes in cancer Biochem Pharmacol. 2019;167:163-172. doi:10.1016/j.bcp.2019.06.004

34. Munnur D, Ahel I. Reversible mono-ADP-ribosylation of DNA breaks. FEBS J. 2017;284(23):4002-4016. doi:10.1111/febs.14297

35. van den Broek E, den Uil S, Coupé V, et al. MACROD2 expression predicts response to 5-FU-based chemotherapy in stage III colon cancer Oncotarget. 2018;9(50):29445-29452. doi:10.18632/oncotarget.25655

36. Van Meter M, Mao Z, Gorbunova V, Seluanov A. Repairing split ends: SIRT6, mono-ADP ribosylation and DNA repair. Aging. 2011;3 (9):829-835. doi:10.18632/aging.100389
37. Golia B, Moeller G, Jankevicius G, et al. ATM induces MacroD2 nuclear export upon DNA damage. Nucleic Acids Res. 2017;45 (1):244-254. doi:10.1093/nar/gkw904

38. Baran B, Mert Ozupek N, Yerli Tetik N, Acar E, Bekcioglu O, Baskin Y. Difference between left-sided and right-sided colorectal cancer: a focused review of literature. Gastroenterol Res. 2018;11 (4):264-273. doi:10.14740/gr1062w

\section{Publish your work in this journal}

Cancer Management and Research is an international, peer-reviewed open access journal focusing on cancer research and the optimal use of preventative and integrated treatment interventions to achieve improved outcomes, enhanced survival and quality of life for the cancer patient.
The manuscript management system is completely online and includes a very quick and fair peer-review system, which is all easy to use. Visit http://www.dovepress.com/testimonials.php to read real quotes from published authors. 